\title{
Innovation Lab: new TRIZ tools for fast idea triggering
}

\author{
Davide Russo ${ }^{1[0000-0001-8000-0147]}$ and Paolo Carrara ${ }^{2[0000-0002-0186-3647]}$ \\ ${ }^{1}$ University of Bergamo, 24044, Dalmine, Italy \\ ${ }^{2}$ Warrant Innovation Lab S.r.l., 42015 Correggio, Italy \\ davide.russo@unibg.it
}

\begin{abstract}
During 2017, University of Bergamo and Warrant Innovation Lab have been starting a partnership to diffuse TRIZ in Italian SMEs. They aim to make SMEs perceive TRIZ as a time-saving methodology, the main needing expressed about innovation. The offering is a one-day problem-solving activity that aims at generating conceptual solutions, potentially patentable. First cases highlighted the reformulation was the trickiest phase: the extraction of the high-level technological alternatives cannot be supported by classical TRIZ tools (object-product transformation, ENV model, IFR, resources, evolutive laws, multiscreen, MTS) for both limited time and lacks in TRIZ skills of the customer. The staff overcomes such a double issue pre-processing the initial problem's information to extract insights from knowledge DBs. The results will be shown as visual-psychological triggers to stimulate the creativity of non-TRIZ-skilled users. The paper will disclose how visual triggers can work and how a triz expert facilitator can create them.
\end{abstract}

Keywords: Visual triggers, Serendipity, Innovation Lab, ENV model.

\section{Introduction}

One of the most barrier to the innovation in SMEs is the lack in time and resources to spend on it [1]. The same is true also for TRIZ, which needs a long training period in order to become an efficient and effective resource. Because of it, the SMEs find it difficult to take advantage of TRIZ.

In SMEs, the incentive to innovate comes from the customers, who ask for improvement in performance or reduction in cost of the products, or from information given by the suppliers, who propose new technologies or products, or from the market competition, which forces toward cost reduction.

Irrespective of the reason for the request, SMEs rely on the insightful-ness, expertise and knowledge of their staff in order to innovate, who usually has traditional designing tools, but lacks in systematic methods specialized in innovation. They tend to outsource the R\&D projects that are burdensome for the internal staff, both in time-consumption and in skills request.

Aiming to support SMEs in the most strategic phases of an innovation project, University of Bergamo (UniBG) and Warrant Innovation Lab (WIL) have been starting a 
partnership since 2017, relying on the Innovation Lab (IL): a consulting activity expressly designed for SMEs and introduced in TFC 2018 [2]. It consists of a one-day team-working session of problem-solving.

To enhance the attitude of the team members, especially for the ones coming from the customer company who are not skilled in TRIZ, the IL exploits visual means to facilitate a serendipity process. They aim to carry the right information to the right person at the right time.

The IL staff can collect in a relatively simple way a large amount of information that are potential activator for serendipity. Furthermore, the people to which propose that information are the customer members of the team. The most difficult step for triggering the serendipity is the choice of the right time to transmit the information.

The visual means exploited by the IL lets the staff to separate in time the information offer from its receiving. Then, the visual means can be elaborated in back-office activity, before the one-day session, while the receiving time should happen during it.

As definition, serendipity means an unplanned, fortunate discovery [3]. It relies on the open-mindedness of the one who is looking for something other, the ability to catch the insight about a potential good idea while its thoughts are focusing on other topics.

In its definition, serendipity hides the overcoming of the psychological inertia.

To facilitate the occurrence of the right time, the staff takes advantage of a revised version of the ENV-model as psychological inertia inhibitor. It forces the company members of the team to leave the comfort-zone about 'what I know to do', calling into question their own problem perception, and going towards the attitude 'what we should be able to do'.

The change of such a perspective let the customer to open their mindedness, overcome the psychological inertia and facilitate the occurrence of the right time for the serendipity.

This paper introduces the way IL exploits to push the serendipity mechanism. In section 2, it illustrates which are the main goals of the customers involved in IL. Section 3 describes how WIL trains the customer participants to the innovation activity. Section 4 shows which are the tools exploited by the IL and which is the workflow of the activity. Then, in Section 5, details about Visual Triggers, how they work and how to prepare them, have been explained.

\section{Customer needs}

Often, SMEs have not employees specifically dedicated to the R\&D who are familiar with inventive problem solving, idea generation or patent literature. If $R \& D$ department exists in SME, in most cases it has not enough time to follow a wide-ranging innovation project.

WIL is a company devoted to facilitating and diffusing the systematic innovation in SMEs, through technology transfer and sharing of knowledge, ideas, technologies and methodologies, especially cooperating with universities and research centers. It offers services to fill in the lacks in innovation methodologies, establishing cooperation and partnership with suitable research teams. 
Thanks to its long-lasting touch keeping with customers, WIL has been getting continuous up-to-date information about their innovation effort. This is the most important requirement to identify the actual goal of an innovation project. Indeed, customer rarely expresses clearly which they are, rather it asks for supporting in the development of an idea and translate it in an innovation project.

Thus, the first marketing activity about IL is understanding which the actual goals of the customer are. WIL offers the IL when it falls in one of main categories below:

1. Often, the customer wants to innovate a product/process for which it is not able to achieve a significant evolutive step. In such a case the customer takes the IL as the opportunity to test TRIZ, like it was a challenge, submitting a real and unsolved problem;

2. Before undertaking a R\&D project, the customer wants to be sure it is going to undertake the less risky innovation path. By the means of the IL, it aims to widen the solution space in order to choose the most suitable conceptual solution for the development;

3. In some cases, especially with start-ups, the customer wants to achieve a new product/process about which it has not proficiency. This is interesting for IL when the customer requires the conceptual design of a new functional solution, that means it asks for inventive problem solving.

The IL was designed in order to save time for the first strategical phases of the innovation process. Nonetheless, it carries other added values.

First, the customer gets a new point of view about its system: tearing down the psychological inertia, IL supports the customer in uncovering new functional solutions, which are original for the reference market.

Widening the solution space, it allows the customer to consider several solving directions in order to plan an innovation and/or patenting strategy. This is particularly felt by start-ups which could get the high-level design of the solution to be developed.

\section{Selection of customer participants}

The IL requires the participants be willing to question their own point of view about the problem and its potential solutions. Further, it is appropriate to have different points of view about the product/process to innovate. Thus, not only technical profiles are required, rather, the presence of marketing employees and decision makers or CEO improves the IL performances.

Due to the long-lasting relationships between WIL and its customers, it enjoys credibility with them. This enables some important aspects for the success of the IL.

First, WIL gives to the customer some indications about the service and how it works. For example, giving them a smattering of TRIZ and/or describing how to difficult is for a specialist to overcome the psychological inertia. Using this opportunity, it can suggest to the customer which are the best employee profiles to involve in the IL. Eventually, it could assist the customer in the choice of people. 
The second aspect is the preparation of the customer participants. Depicting the specific feature of the service is a way to prepare them for the work they will do with UniBG. They get upfront what to expect by the one-day innovation activity

Lastly, together with what just said, the credibility of WIL with the customer is implicitly transferred to UniBG. This eases a lot the first phase of the IL, when the customer participants must be convinced about the goodness of the methodology behind the service.

\section{Tools and workflow}

In the morning session of IL, the participants work on generalizing the problem and enlarging the space of its solutions. The TRIZ expert guides the participants along an innovation path [2] that include techniques of abstraction of the target product/process (functional modelling), suppression of the constraints (modified ENV model [4]), technological alternatives analysis (ENV model integrating the IFR [5] and IR tools specifically developed), management of requirements (market potential [6, 7]) and overcoming of psychological inertia (visual triggers).

In the afternoon session of IL, the participants choose the most interesting solving direction and develop it. The staff guides them exploiting the knowledge about physical contradictions, separation principles and other classic TRIZ tools, without explicitly describe them to the participants.

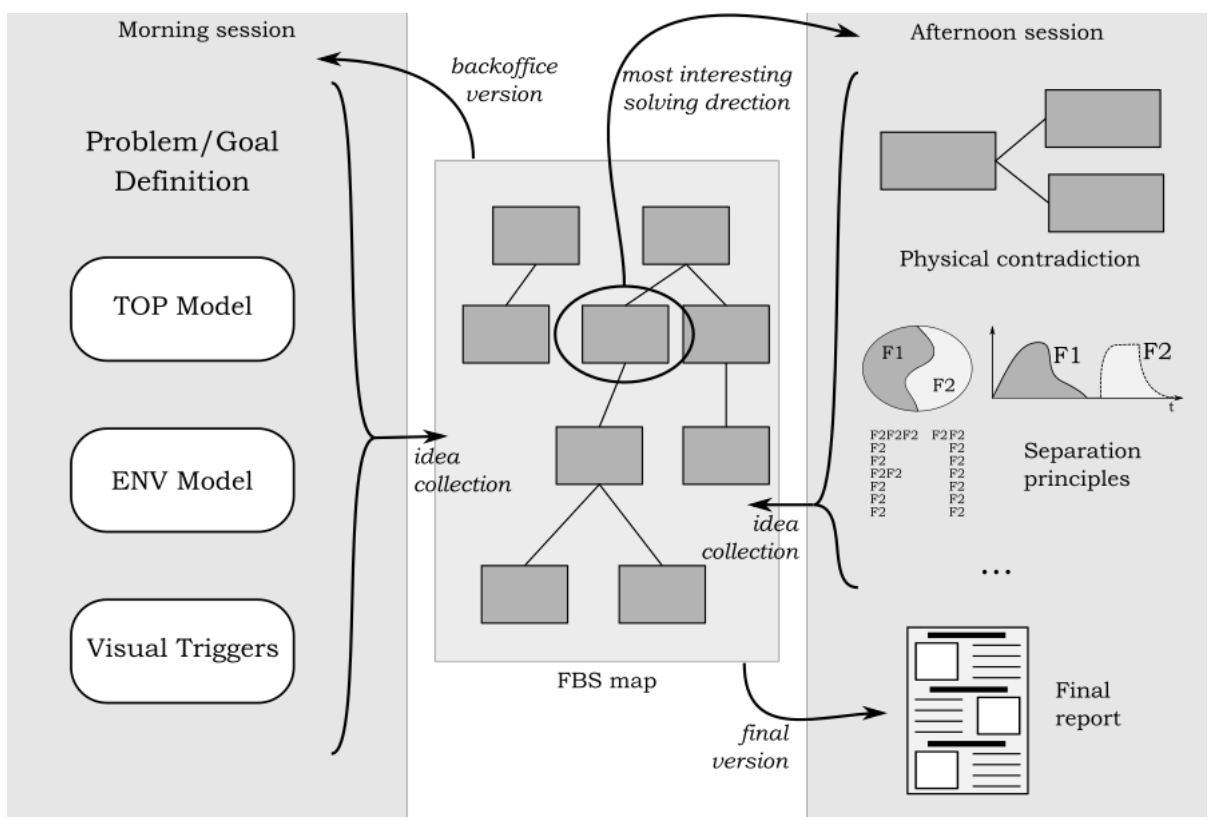

Fig. 1. workflow and tools exploited by the IL. All interesting ideas are collected in the FBS map 
The ideas generated are tracked and organized according to the FBS ontology [8] on a map collecting high-level functional solutions (FBS map). Fig. 1 shows the workflow of IL, the main tools exploited by the staff to lead the innovation path and how the FBS map works during the day.

More details about Visual Triggers and how to populate the FBS map are going to explain in next chapters.

\section{$5 \quad$ Visual Triggers}

Serendipity means an unplanned, fortunate discovery [3]. It is a common occurrence throughout the history of product invention and scientific discovery. The term is often applied to inventions made by chance rather than intent.

The goal of the IL staff is to create 'a bump in the road', so as to guide the accidental knowledge.

Many of the most well-known mechanisms for conducting a good problem solving path are those related to the sense of belonging to the problem: it is easier to passionately conduct a problem that is close to our heart, or a direction discovered by us rather than committing ourselves carry out the task assigned or discovered by someone else.

For this reason, the inventive idea must come from the customer experts and never from the staff! Even better when all the participants reach the idea at the same time, or if they are all aware that they could have grasped it by themselves.

It would be good whether nobody thought that an expert has dropped none of the ideas that come along. The 'wow effect' created by facilitators is one of the worst mistakes that can be made in this activity.

On the contrary, all the participants must become actors, and to do this it is necessary to find an effective mechanism of accidental knowledge, capable of igniting the mysterious mechanism of serendipity.

Accidental knowledge means that knowledge that is acquired in a completely random way, through what is called the knowledge accident. This terminology belongs to that discipline called knowledge management, which studies how it is possible to ensure that "the right knowledge reaches the right person at the right time to be able to make the best possible decision".

Specifically, the knowledge accident represents a mechanism capable of conveying the right knowledge to the right person, not necessarily at the right time. This delay is an aspect that can completely frustrate the transfer of this knowledge.

Better to give the right information without the user having the perception of being guided in the choice, nor to arrive at the solution 'prepared' already by the staff.

In order to simulate accidental knowledge, a set of images, called visual triggers is prepared.

\subsection{Triggers functioning}

The Images are designed to evoke functions or behaviors useful for identifying an alternative way for solving the initial problem. 
According to FBS theory [8] a solution can be conceived as a set of elements (Structure) working together in order to realize a Behavior that allows the structure reaching a goal (Function).

Visual triggers are images of products, that being outside of our problem context, do not evoke structure analogies but only behavioral or functional alternatives.

They must be general enough to allow the user the freedom to decline it in the way he intends to use it for his own purposes.

For example, in a recent case, it was necessary to replace a cutting blade positioned with extreme precision inside a very heavy chassis. The changeover operation is frequent due to the high wear on the blade and every time an important machine stop is required with a very well-prepared intervention by the workers.

All the products sold by the company involved use the same maintenance process, which includes a "book" machine opening device to facilitate operator entry into the machine.

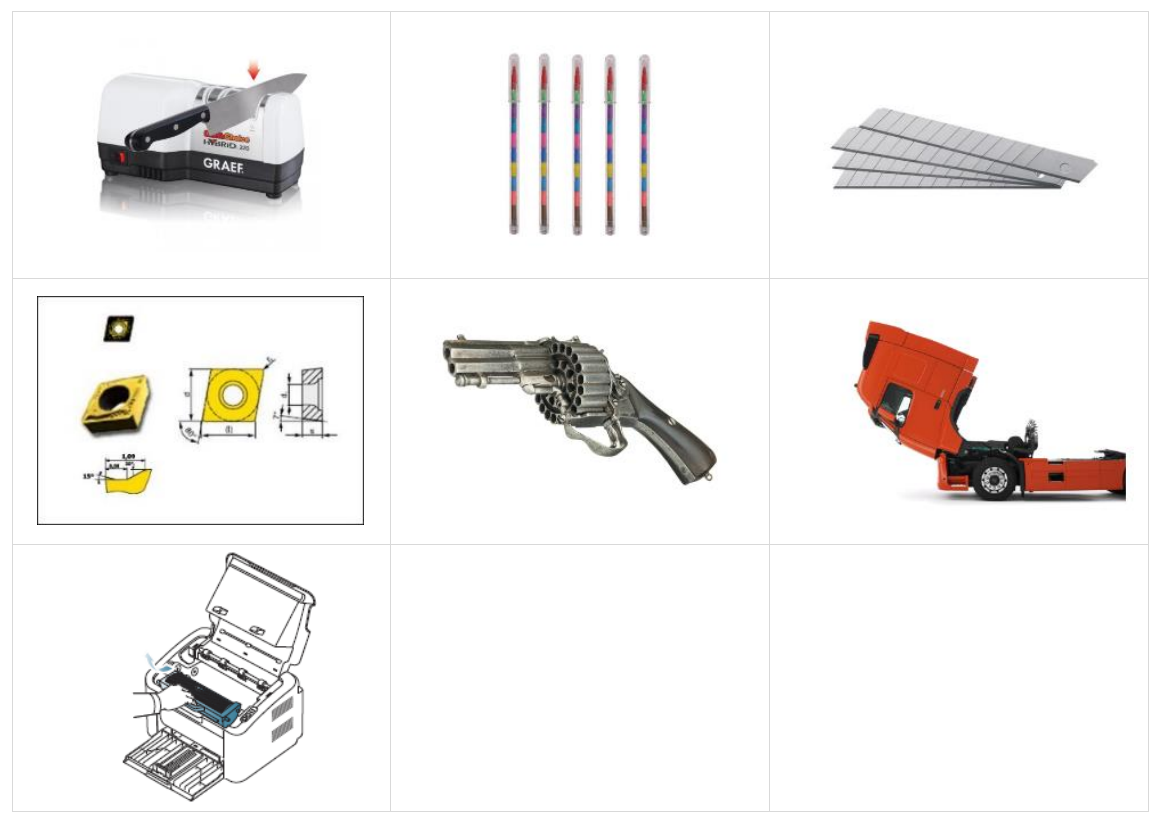

Fig. 2. Visual triggers at functional level, explain strategies for replace/restore the blade

Visual triggers (shown in Fig. 2) help remove this type of condition, moving the problem to other areas.

For example, the first image is designed to suggest that you might not have to replace the blade in case someone is able to restore the thread. It may come out of refining methods or simply the idea of not having it sharpened anymore because it sharpens itself, or a blade that never loses the thread either because it has a much longer life, or because the system removes the condition so the blade loses the thread. 
The second image indicates an alternative way of replacing things, restoring the blade with a ready one according to a precise and preset dynamic.

In a like manner, the cutter blades, the third visual trigger, produce a similar path but with completely different dynamics. Sometimes it is the union of several triggers that trigger the creative mechanism.

In the case of the cutting edges of image 4 we have tried to explain how each blade has multiple profiles that can be used as cutting edges, instead of always putting a new one and having to recalibrate the system you could try to understand how to modify the housing for offer a new cutting edge. This image suggests using the same blade in a new position but does not say how.

Contrarywise, the subsequent images give us two different ways of preparing the new blade, respectively rotating or reversing.

The last case is the toner of the printer, which indicates a different way of inserting an object.

In the next figure we show an example of triggers focused on the goal of blocking. In them we suggest alternative ways at the level of Behavior.

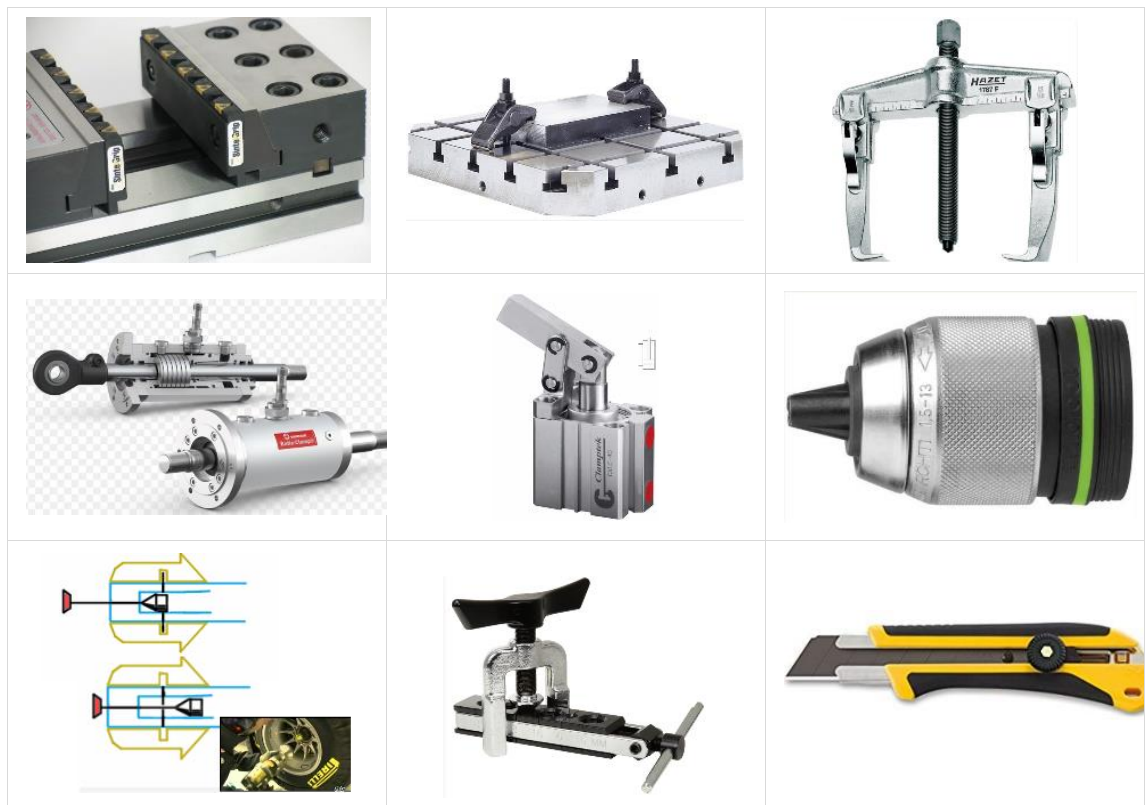

Fig. 3. Visual Triggers at behavioral level, explaining different ways of blocking something

As can be seen, the evocative level of these triggers is much more limited than in the previous case; the creative effort required of the user is undoubtedly much shorter. For each option the effort is only to imagine how to contextualize the solution to one's own field, assessing its technical feasibility and side effects. 
What is shown in the Fig. 3 is a list of alternatives that could be generated automatically by a knowledge search tool.

The higher the degree of specialization of the triggers, the greater the possibility of automating the automatic content search process.

Similarly we look at the search for all the alternative ways in which a function can be exploited by exploring the various associated physical effects [9].

\section{$5.2 \quad$ Triggers preparation}

For the construction of visual triggers, it is necessary to know very well all TRIZ tools. The most suitable tool for this purpose is the ENV model.

According to the OTSM TRIZ theory of Nikolai Khomenko [4], ENV model helps to describe one or more functions starting from its decomposition in features.

For each parameter/feature its value is described indicating to which element it is referenced. The function is defined as the variation of the feature value during the transformation induced by the function, before and after.

In this way it is possible to describe the main function of a system through a list of transformations, expressed as a variation of the value of the technical parameters.

To this, we can also add further information with respect to the value of the characteristic in the ideal situation.

The comparison between expected value (ideal) and obtained value (real) determines in the user an inventive trigger to improve the starting system.

The basic idea for the construction of the visual triggers is to work ahead of the day, applying this tool and defining an image for each suggested transformation, avoiding images taken from the context in which you are operating.

The choice of images is based on the number of constraints that can be removed during the day with the customer, as well as the number of unpublished prospects with which to address the problem.

The basic idea is to start from the main transformation of the system, imagining how the object of the main function is transformed into a desired product, and from here to hypothesize at a high level the alternative and integrative functions that could arise during the session, masking them with images that subliminally call the concept back without explicitly mentioning it.

The purpose of the triggers is to investigate possible alternatives at the function level. Once found, they must be hierarchically organized according to the following model (see Fig. 4). Once the map of alternatives is built, the facilitator can apply the other tools of the TRIZ methodology to formulate questions that push the experts of the sector to find further alternatives. IFR, multiscreen works very well both at the level of $\mathrm{F}$ and B. 


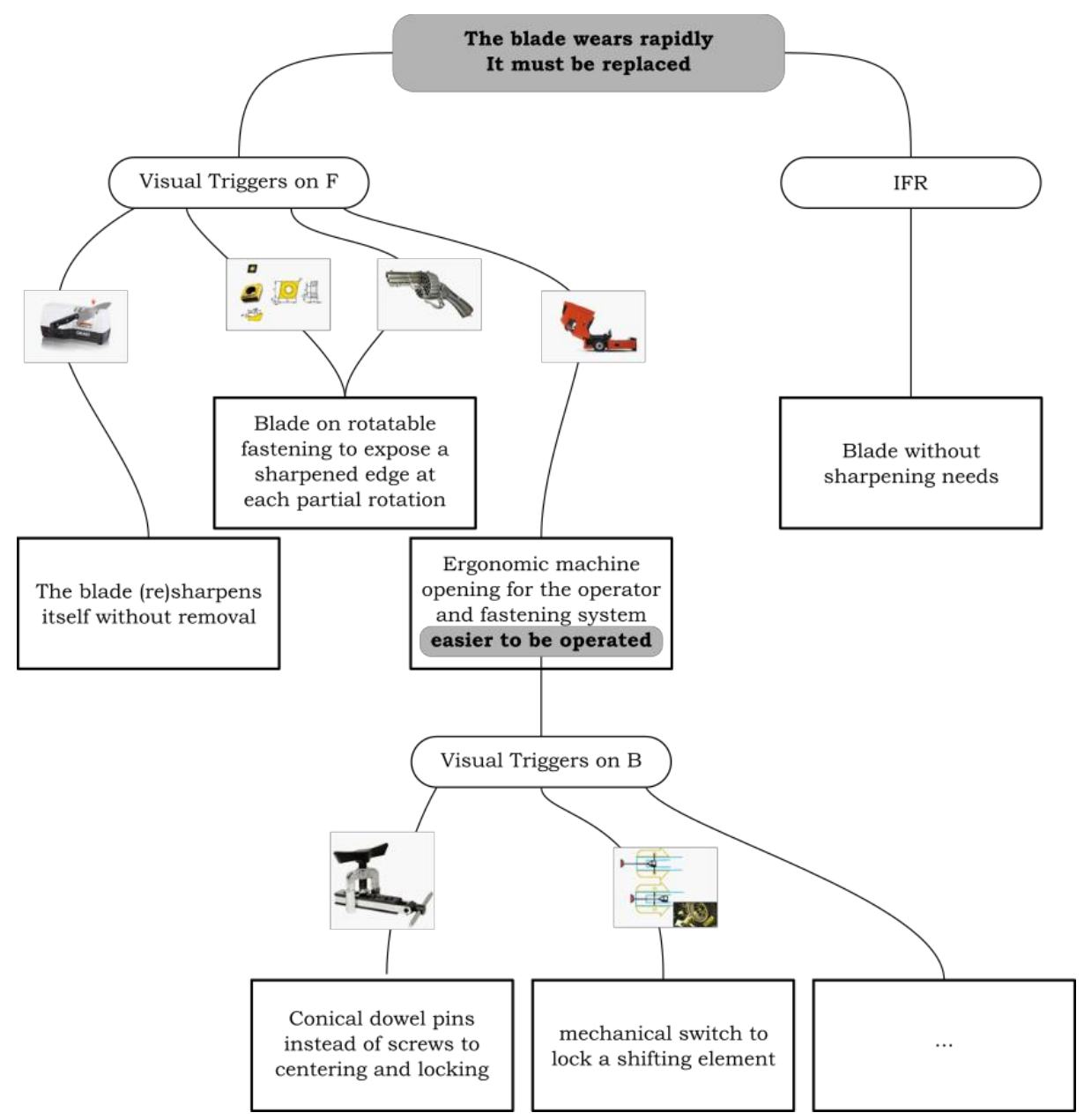

Fig. 4. Example of the hierarchical map of alternatives coming from F-level and B-level triggers and IFR

\section{Conclusion}

Warrant Innovation Lab and University of Bergamo have been starting a partnership that aims to support SMEs in the most strategic phases of their innovation projects limiting the time request to generate conceptual solutions. They offer the Innovation Lab, which is a TRIZ-based problem-solving activity that lasts one day and aims at generating conceptual solutions, potentially patentable.

The Innovation Lab adjusts the TRIZ-based innovation path to reach at least a conceptual solution within the limited time available. Especially, it exploits Visual Trig- 
gers, which are images representing functional examples able to convey insights in implicit manner. Thanks to this approach, the staff facilitates the knowledge accident, the idea generation mechanism behind serendipity.

The customers responded positively to the service, expressing satisfaction about the approach and results. Later to the Innovation Lab, all of them have been carring forward the innovation project. The knowledge uncover by the Innovation Lab allows them to save time also in the following development steps of the solution.

Irrespective to what initial attitude the customer had regarding the opportunity to patent the solution, all cases to date faced gave rise to a patent application. This proves even more the results are interesting and valuable for the customers.

Despite the advantages taken from the conceptual solutions the Innovation Lab allowed to grasp, the customers did not show interest in deepening their knowledge about TRIZ, for example through participating to learning or training courses. Rather, they consider the Innovation Lab the way to solve an inventive problem they are not able to overcome.

Of course, this is a positive result, but further developments on the Innovation Lab and on the approach to the customers must be considered to increase the interest about TRIZ.

\section{References}

1. Russo D, Regazzoni D, Rizzi C (2018) A Long-Term Strategy to Spread TRIZ in SMEs. Analysis of Bergamo's Experience. In: Advances and Impacts of the Theory of Inventive Problem Solving. Springer International Publishing, Cham, pp 147-157

2. Russo D, Carrara P, Marusi M (2018) Innovation Lab: How to Generate Patents in One Day. In: Cavallucci D, de Guio R, Koziolek S (eds) TRIZ Future Conference 2018, Professional Proceedings

3. serendipity | Definition of serendipity in English by Oxford Dictionaries. https://en.oxforddictionaries.com/definition/serendipity. Accessed 11 May 2019

4. Cavallucci D, Khomenko N (2007) From TRIZ to OTSM-TRIZ: addressing complexity challenges in inventive design. Int J Prod Dev 4:4 . doi: 10.1504/IJPD.2007.011530

5. Altshuller G (2005) The Innovation Algorithm - TRIZ, systematic innovation and technical creativity (Translated by Shulyak, L. \& Rodman, S.)

6. Livotov P (2015) Using Patent Information for Identification of New Product Features with High Market Potential. Procedia Eng 131:1157-1164 . doi: 10.1016/j.proeng.2015.12.438

7. Livotov P (2008) Method for Quantitative Evaluation of Innovation Tasks for Technical Systems, Products and Processes. In: Proceedings of ETRIA World Conference "Synthesis in Innovation." pp 197-199

8. Gero JS, Kannengiesser U (2004) The situated function-behaviour-structure framework. Des Stud 25:373-391 . doi: 10.1016/j.destud.2003.10.010

9. Montecchi T, Russo D (2015) FBOS: Function/Behaviour-Oriented Search. Procedia Eng 131:140-149 . doi: 10.1016/j.proeng.2015.12.363 